\title{
Detecting hepatocellular carcinoma in blood
}

\author{
Cell Research (2015) 25:1279-1280. doi:10.1038/cr.2015.136; published online 24 November 2015
}

Liquid biopsy is ideal for early diagnosis of cancer and for prognosis upon treatment. Wen et al. describe a methylated CpG tandems amplification and sequencing method to profile hypermethylated $\mathrm{CpG}$ islands genome-widely in cell-free DNA, and further identify high performance markers in blood for potential detection of early stage hepatocellular carcinoma.

Early diagnosis is key to cancer prevention and treatment. When physiological consequences of cancer are observed it could be too late for the optimal treatment and therapy [1]. Traditional biopsy has been widely used for diagnosis; however, it is difficult to frequently perform biopsy. In many cases it is impossible to perform biopsy of solid tumors grown in deep tissues. Cell-free nucleic acids (cfNAs) offer an alternative option. The presence of cfNAs in blood was described in 1948. However, cfNAs such as DNA, mRNA and microRNAs (miRNAs) were not recognized as potential disease biomarkers until recently because of the rapid advance of sequencing technologies [2-4]. The apoptosis and necrosis of tumor tissues can lead to release of cell-free DNAs (cfDNAs) into the circulating system [5]; these cfDNAs contain crucial genetic and epigenetic information for early diagnosis if sensitive and accurate methods can be developed.

Human hepatocellular cancer, one of the most lethal cancers, is characterized by progressive accumulation of epigenetic changes [6], among which hypermethylation of cancer-associated DNA offers distinct markers for diagnosis. DNA methylation patterns could change throughout the cancer development stages. If the same DNA methylation changes could be monitored in cfDNA released by tumor one could trace the emergence of the cancer, monitor the progression, and predict effects of treatments. Despite these advantages, current cfDNA detection is significantly hampered by the lack of sensitivity because only a very small amount of cfDNA could be obtained from plasma and serum. cfDNA is also heavily fragmented (between 200 400 bp), adding additional challenges.

Faced with these challenges, Wen et al. [7] invent methylated $\mathrm{CpG}$ tandems amplification and sequencing (MCTASeq), a method that takes advantage of the fact that $\mathrm{CpG}$ tandems are highly enriched in the $\mathrm{CpG}$ island-containing promoters of human genome. These CpGs are typically unmethylated but tend to gain hypermethylation in hepatocellular carcinoma (HCC) [6]. The cfDNAs released into circulation carry the same hypermethylation patterns, thereby providing accurate information of the presence of HCC in patients. In their new method, cfDNA is treated with bisulfite, during which non-methylated $\mathrm{C}$ (cytosine) is converted to $\mathrm{U}$ (uracil) while methylated $\mathrm{C}$ remains unaffected. They then use a pair of primers to specifically amplify DNA loci that contain hypermethylated CGCGCGG, a sequence frequently presented in $\mathrm{CpG}$

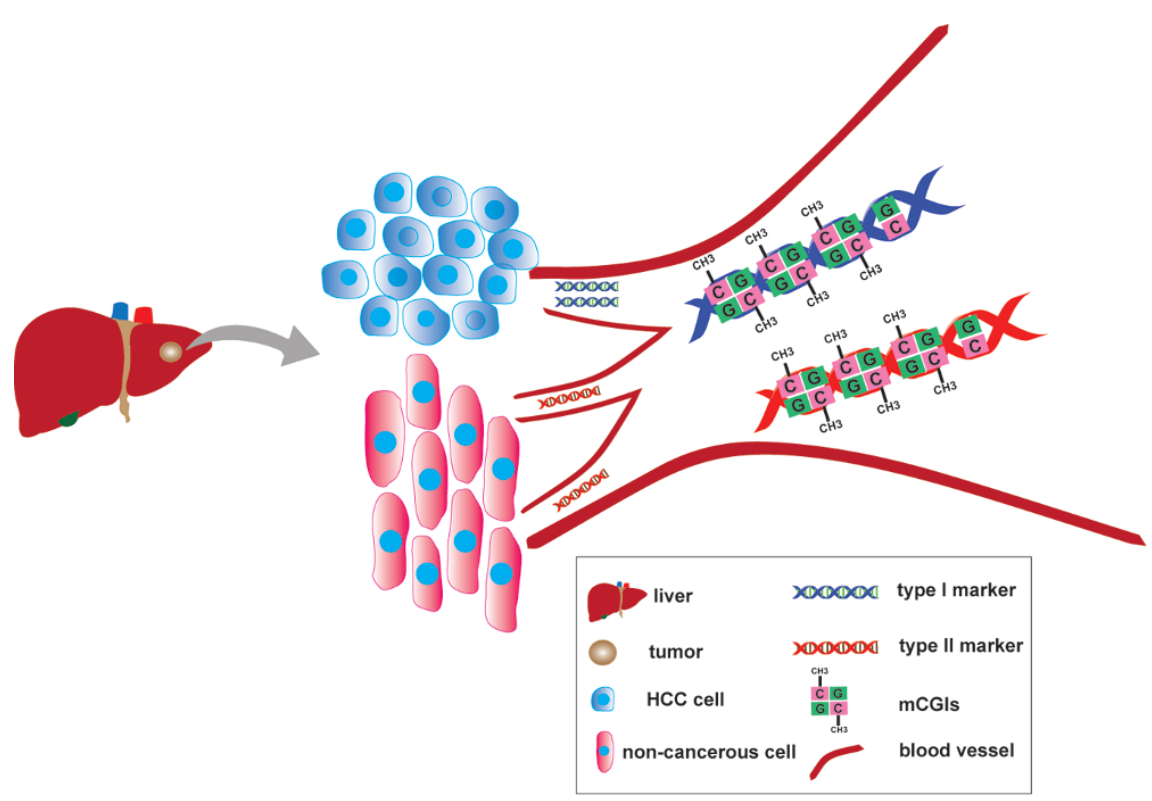

Figure 1 Hypermethylated cfDNA released into the blood can be detected with a new method. Cell-free DNA-containing hypermethylated CpG islands (mCGls) circulating in the blood of heptocellular carcinoma patients can be detected for early diagnosis. These marker DNAs are released by either tumor cells undergoing apoptosis or necrosis (type I) or adjacent non-cancerous cells affected by tumor growth (type II). 
islands and tend to be methylated in cancer tissues. The focus on the CGCGCGG-containing loci may miss other potential markers; however, it offers the sensitivity required for methylation detection in cfDNA. Validation data of MCTA-Seq shows that it is highly reproducible and sensitive, with the detection limit down to as low as 7.5 pg ( 2.5 haploid genome equivalents). Existing biomarkers that are frequently hypermethylated in human cancers [8], such as VIM, SEPT9, NDRG2 and RASSF1 [8], could be detected with high sensitivity by using MCTA-Seq. The method, although limited by the requirement of the CGCGCGG sequence content, is genome-wide and offers sufficient information about $\mathrm{CpG}$ island methylation changes in HCC.

Wen et al. applied the new method to detect tumor-specific CGI methylation with plasma samples from HCC pa- tients, cirrhosis patients, and normal individuals. Two types of biomarkers have been identified for early stage HCC diagnosis (Figure 1). Type I markers possess significantly higher methylated CGIs than cancer-free individuals. Type II markers are tissue-specifically methylated CGIs, which tend to be restricted to liver cells under normal circumstances but are released into the blood when malignance occurs. Type II markers dominate in the cfDNA at early stage of HCCs, making them sensitive signs of tumor emergence.

The new method and the use of marker combination shown by Wen et al. provide a new strategy for DNA methylation detection from cfDNA. It may have widely applicable potential not only in HCC but also a cohort of other cancer types.

Lulu $\mathrm{Hu}^{1,2}$, Chuan $\mathrm{He}^{1,2}$
${ }^{1}$ Department of Chemistry, Department of Biochemistry and Molecular Biology, and Institute for Biophysical Dynamics, The University of Chicago, Chicago, IL 60637, USA; ${ }^{2}$ Howard Hughes Medical Institute, The University of Chicago, Chicago, IL 60637, USA

Correspondence: Chuan $\mathrm{He}$

E-mail: chuanhe@uchicago.edu

\section{References}

1 Bettegowda C, Sausen M, Leary RJ, et al. Sci Transl Med 2014; 6:224ra24.

2 Sorenson GD, Pribish DM, Valone FH, et al. Cancer Epidemiol Biomarkers Prev 1994; 3:67-71.

3 Vasioukhin V, Anker P, Maurice P, et al. Br J Haematol 1994; 86:774-779.

4 Kaiser J. Science 2010; 327:1074.

5 Schwarzenbach H, Hoon DS, Pantel K. Nat Rev Cancer 2011; 11:426-437.

6 Pogribny IP, Rusyn I. Cancer Lett 2014; 342:223-230.

7 Wen L, Li J, Guo H, et al. Cell Res 2015; 25:1250-1264.

8 Lofton-Day C, Model F, Devos T, et al. Clin Chem 2008; 54:414-423. 